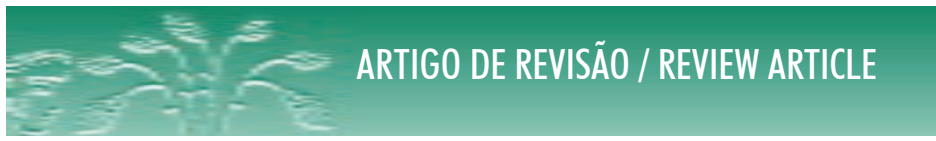

DOI 10.12957/demetra.2014.6670

\title{
Programas de redução de peso via web: uma revisão
}

\section{Internet-based weight loss programs: a review}

\author{
Camila Ragne Torreglosal \\ Andréa Polo Galante ${ }^{2}$ \\ Alexandre Rodrigues Lobo ${ }^{3}$ \\ Célia Colli ${ }^{3}$ \\ ${ }^{1}$ Curso de Pós-graduação em Nutrição Humana \\ Aplicada. Universidade de São Paulo. São Paulo, \\ SP, Brasil. \\ ${ }^{2}$ Centro Universitário São Camilo. São Paulo, \\ SP, Brasil. \\ ${ }^{3}$ Faculdade de Ciências Farmacêuticas, \\ Departamento de Alimentos e Nutrição \\ Experimental. Universidade de São Paulo. São \\ Paulo, SP, Brasil. \\ Correspondência / Correspondence \\ Camila Ragne Torreglosa \\ E-mail: camilatorreglosa@usp.br
}

\section{Resumo}

Introdução: A prevalência de obesidade na população adulta é uma preocupação em saúde pública. As terapias consensuais são farmacológicas, cirúrgicas e comportamentais, mas quando não associadas a mudanças dos hábitos de vida, costumam ser ineficientes em longo prazo. Programas para redução de peso via web são uma alternativa complementar. Objetivo: Relatar trabalhos de intervenção alimentar via web, analisar o modelo de intervenção utilizado e os resultados obtidos na redução do peso. Métodos: Foram selecionados apenas ensaios clínicos randomizados e controlados sobre intervenção nutricional aplicada via web publicados entre os anos de 2002 e 2012, encontrados nos bancos de dados PubMed via MEDLINE e Web of Science. Os artigos selecionados foram avaliados por categorias, sendo Categoria 1: objetivos, grupo estudado, tamanho da amostra, tempo e perda de seguimento; e Categoria 2: desenho da intervenção, referencial teórico, estratégias de personalização e desfechos. Resultados: 15 ensaios clínicos randomizados foram selecionados. As estratégias de personalização foram elaboradas a partir de informações coletadas por meio de questionários preenchidos pelos usuários dos programas. As intervenções utilizaram modelos baseados em teorias do comportamento. A redução no peso corporal variou entre 0,9 a 8,8 Kg. Conclusão: Os programas para redução do peso via web proporcionam interação com o usuário e em alguns casos promovem a redução do peso. Contudo, os recursos utilizados não são descritos detalhadamente pelos autores, o que dificulta a criação de novos programas embasados cientificamente.

Palavras-chave: Dieta. Obesidade. Perda de Peso. Educação Alimentar e Nutricional. Internet. Comportamento Alimentar. Estudos de Intervenção. 


\section{Abstract}

Introduction: The prevalence of obesity in the adult population is a public health concern. Pharmacological, surgical and behavioral therapies are consensual, but when they are not associated with changes in lifestyle are often ineffective in the long term. Web programs for weight loss are an alternative and complementary. Objective: To report web interventions for weight loss, analyze the model of intervention and results in weight reduction. Methods: Only randomized clinical trials of nutritional intervention applied via web published between the years 2002 and 2012 were selected. Databases were MEDLINE via PubMed and Web of Science. The articles were evaluated by categories, being Category 1: objectives, study group, sample size, time, and loss of follow up; and Category 2: intervention design, theoretical framework, personalization strategies and outcomes. Results: 15 randomized controlled trials were selected. The personalization strategies have been prepared from information collected through questionnaires filled by the users of programs. The interventions used models based on theories of behavior. Reduction in body weight achieved in the web groups ranged from 0.9 to $8.8 \mathrm{Kg}$. Conclusion: Web programs for weight loss provide interaction among users and in some cases promotes weight reduction. However, the resources used are not described in detail by the authors, and this hinders the creation of new science-based programs.

Key words: Diet. Obesity. Weight Loss. Food and Nutrition Education. Internet. Food Behavior. Intervention Studies.

\section{Introdução}

O aumento da prevalência de sobrepeso e obesidade na população adulta é uma preocupação em saúde pública. ${ }^{1}$ Estimativas da Organização Mundial da Saúde apontaram que, em 2008, 1,4 bilhão de indivíduos com 20 anos ou mais de idade no mundo apresentaram sobrepeso e, aproximadamente, 500 milhões de adultos eram obesos (IMC $\geq 30 \mathrm{~kg} / \mathrm{m}^{2}$ ). ${ }^{2}$

No Brasil, dados de inquéritos populacionais revelaram que, em 2011, o excesso de peso esteve presente em, no mínimo, 39,8\% da população de São Luís e em 55,4\% na de Porto Alegre. ${ }^{3}$

O aumento progressivo do índice de massa corporal está relacionado ao surgimento das doenças crônicas não transmissíveis, como as cardíacas, o acidente vascular cerebral, o diabetes e o câncer. Essas doenças são a causa de óbitos de 2,8 milhões de indivíduos no mundo a cada ano. ${ }^{4}$ 
O peso corporal é determinado pela interação de fatores genéticos, ambientais, psicológicos e sociais, e o excesso de peso ocorre quando há desequilíbrio entre esses fatores. ${ }^{5}$

Muitos especialistas associam tal desequilíbrio ao estilo de vida contemporâneo, que é influenciado por aspectos como crescimento econômico, industrialização e mecanização da produção de alimentos, urbanização, maior acesso a alimentos em geral e globalização. A presença desses fatores faz com que o tratamento da obesidade seja complexo e exija o envolvimento de uma equipe multiprofissional. Por isso, é importante tratar as causas relacionadas ao ambiente e às mudanças no estilo de vida..$^{6-8}$

As terapias consensuais indicadas para o tratamento incluem as farmacológicas, a cirúrgica e a comportamental. As duas primeiras, quando não são associadas a mudanças dos hábitos de vida, podem ser ineficientes no longo prazo. A alteração do estilo de vida refere-se à adequação dos hábitos relacionados à alimentação inadequada, ao sedentarismo e ao estresse. ${ }^{8}$

Estudos que focaram a mudança do estilo de vida demonstraram bons resultados na diminuição do peso corporal para níveis clinicamente significativos (redução de 5 a $10 \%$ do peso atual). ${ }^{9}$ Um exemplo é o Diabetes Prevention Program, intervenção conduzida por equipes compostas por nutricionistas, médicos e psicólogos, que visa à mudança do comportamento alimentar e que reduziu em cerca de $60 \%$ o risco de desenvolver diabetes. Metade dos indivíduos participantes diminuíram em $7 \%$ o peso corporal inicial em um período de seis meses de tratamento. ${ }^{10}$ Apesar dos benefícios, intervenções como esta são de alto custo, pois incluem gastos diretos com transporte, profissionais de saúde e estrutura de atendimento. Além disso, não atingem um grande número de indivíduos. ${ }^{11}$

Nos últimos dez anos, os programas intervencionistas para redução de peso via internet apresentam-se como alternativas mais acessíveis por terem menor custo e complementarem as ações nutricionais presenciais. ${ }^{12}$ Revisões sistemáticas da literatura mostraram evidências científicas relacionadas à efetividade dos programas educacionais via web na educação nutricional e na redução e manutenção do peso corporal..$^{13,14}$

A comunicação com o indivíduo via web se estabelece por meio de sistemas que possibilitam tratá-lo em tempo real, de forma personalizada e interativa. Dessa forma, a redução do peso e a reeducação nutricional consideram as preferências e necessidades individuais. ${ }^{15-18}$ Essa personalização é definida como tailoring, em que são utilizadas estratégias educativas baseadas no comportamento humano para estabelecer metas individuais, procurando melhorar a adesão às orientações. ${ }^{15-18}$

A oferta de informações e programas para redução do peso na web é grande. Uma busca no sítio eletrônico Google, utilizando as palavras entre aspas "emagrecimento online", resultou em 9.130 páginas com assuntos relacionados ao tema. 
No Brasil, 45\% da população possuem computador na residência e 38\% destes estão conectados à internet. ${ }^{19}$ Todavia, na literatura brasileira, existem poucos trabalhos que exploram essa temática, e não foi encontrada referência que explorasse os modelos de intervenção para redução de peso utilizados em programas via web. ${ }^{20}$

Este estudo é uma revisão de literatura que tem como objetivos explorar estudos de intervenção alimentar via web, analisar os modelos de intervenção utilizados e os resultados obtidos pelos indivíduos que acessam esses programas.

\section{Métodos}

Para realizar a busca dos artigos científicos, foi delimitado o período de 2002 a 2012. Os bancos de dados utilizados foram o Pubmed via MEDLINE e o Web of Science (ISI). Os termos utilizados para a busca foram: internet, overweight, obesity, body weight changes, food habits, web-based, computer tailoring, nutrition education e adults. A busca booleana seguiu a formulação: (internet $O R$ web-based OR computer tailoring) AND (overweight OR obesity) AND (body weight changes OR food habits OR nutrition education) AND (adults).

Artigos nos idiomas português e inglês publicados em revistas científicas indexadas sobre medicina preventiva, medicina e internet, educação e comportamento em saúde e obesidade foram selecionados. A palavra tailoring neste artigo foi traduzida como "personalização".

Foram considerados critérios de inclusão dos artigos: ensaios clínicos randomizados e controlados sobre intervenção nutricional aplicada via computador de forma exclusiva ou mista e com desfechos que tratassem sobre comportamento alimentar e peso corporal foram incluídos. Foram excluídos os estudos que não utilizaram no grupo intervenção a internet como forma principal de orientação do participante - por exemplo, aqueles que utilizaram programas elaborados em $C D-R O M$. E ainda, artigos sobre o desenho do estudo.

\section{Seleção dos artigos}

Os artigos selecionados foram avaliados por categorias, de acordo com as seguintes questões de pesquisa:

- Categoria 1: Desenho do estudo, objetivos, grupo estudado, tamanho da amostra, tempo e perda de seguimento

1. Qual é o objetivo principal do estudo?

2. Quem é o grupo estudado por sexo, idade e índice de massa corporal médios? 
3. Qual é o tamanho da amostra?

4. Qual é o tempo de seguimento do estudo?

5. Qual é a perda de seguimento?

- Categoria 2: Desenho da intervenção e grupo controle, referencial teórico, estratégias de personalização e desfechos

6. Qual é o desenho de estudo dos grupos intervenção e controle?

7. Qual é o referencial teórico comportamental utilizado no grupo intervenção para redução de peso?

8. Quais são os mecanismos ou estratégias de personalização (tailoring)?

9. Quais são os desfechos relacionados com redução de peso (em kg de peso) e redução clinicamente significativa de peso $(<5 \%)$ ?

\section{Resultados}

A busca resultou em 201 artigos, dos quais 15 ensaios clínicos randomizados atingiram os critérios de inclusão e foram selecionados para análise. Dentre os estudos, dez foram desenvolvidos nos Estados Unidos, três na Austrália, ima na Inglaterra e um na Holanda.

Categoria 1: Desenho do estudo, objetivos, grupo estudado, tamanho da amostra, tempo e perda de seguimento (tabela 1)

Com relação ao sexo, os estudos de Webber et al. ${ }^{21}$ e Mouttapa et al. ${ }^{22}$ tiveram foco específico no público feminino, e os estudos de Morgan et al. ${ }^{23}$ e Patrick et al., ${ }^{24}$ no sexo masculino. Nas demais pesquisas, não houve subdivisão em relação a essa característica.

A média do índice de massa corporal esteve em obesidade classe 1 (de 30 a 34,9kg) em 78\% dos estudos. A faixa etária variou entre 35,9 e 50 anos. Os números mínimo e máximo de participantes foram 51 pessoas no estudo de Pellegrini et al. ${ }^{25} \mathrm{e} 2.862 \mathrm{em}$ Rothert et al. ${ }^{26} \mathrm{O}$ tempo de seguimento esteve entre dois e 12 meses, sendo que em 33\% dos estudos os pacientes foram acompanhados por seis meses.

A maioria dos estudos (60\%) apresentou perda de seguimento superior a 20\%. ${ }^{23,24,26-31}$ Alguns estudos identificaram que a perda de seguimento esteve relacionada com a baixa resposta na 
redução do peso corporal, a preferência por atendimento presencial e com o fato de que a intervenção via web não foi suficientemente personalizada. Apenas seis estudos (40\%) apresentaram perda inferior a $20 \% .{ }^{21,22,32-35}$

Tabela 1. Sexo, índice de massa corporal médio, idade média, tamanho da amostra, tempo e perda de seguimento. São Paulo-SP, 2012.

\begin{tabular}{|c|c|c|c|c|c|c|}
\hline Autores & Sexo & $\begin{array}{c}\text { IMC } \\
\text { médio } \\
(\mathrm{kg} / \mathrm{m} 2)\end{array}$ & $\begin{array}{l}\text { Idade } \\
\text { média }\end{array}$ & $\begin{array}{l}\text { Tamanho } \\
\text { da amostra }\end{array}$ & $\begin{array}{c}\text { Tempo de } \\
\text { seguimento } \\
\text { (meses) }\end{array}$ & $\begin{array}{c}\text { Perda de } \\
\text { seguimento } \\
(\%)\end{array}$ \\
\hline $\begin{array}{l}\text { Mc Connon } \\
\text { et } a l .{ }^{27}\end{array}$ & $\begin{array}{l}\text { Homens } \\
\text { e } \\
\text { Mulheres }\end{array}$ & $*$ & $*$ & 221 & 12 & 41 \\
\hline $\begin{array}{l}\text { Morgan et } \\
a_{l .}{ }^{23}\end{array}$ & Homens & $30,6(2,8)$ & $\begin{array}{c}35,9 \\
(11,1)\end{array}$ & 65 & 12 & 29 \\
\hline $\begin{array}{c}\text { Harvey- } \\
\text { Berino, et } \\
\text { al. }{ }^{32}\end{array}$ & $\begin{array}{l}\text { Homens } \\
\text { e } \\
\text { Mulheres }\end{array}$ & $35,7(5,6)$ & $\begin{array}{l}46,6 \\
(9,9)\end{array}$ & 481 & 6 & 4 \\
\hline $\begin{array}{c}\text { Webber, et } \\
a l .{ }^{21}\end{array}$ & Mulheres & $31,1(3,7)$ & $50(9,9)$ & 66 & 4 & 2 \\
\hline $\begin{array}{l}\text { Polzien, et } \\
\text { al. }{ }^{33}\end{array}$ & $\begin{array}{l}\text { Homens } \\
\text { e } \\
\text { Mulheres }\end{array}$ & $33,1(2,8)$ & $\begin{array}{l}41,3 \\
(8,7)\end{array}$ & 57 & 3 & 0 \\
\hline Gold, et al. ${ }^{28}$ & $\begin{array}{l}\text { Homens } \\
\text { e } \\
\text { Mulheres }\end{array}$ & $32,3(3,9)$ & $\begin{array}{c}46,5 \\
(10,7)\end{array}$ & 126 & 12 & 35 \\
\hline $\begin{array}{c}\text { Rothert, et } \\
a l .{ }^{26}\end{array}$ & $\begin{array}{l}\text { Homens } \\
\text { e } \\
\text { Mulheres }\end{array}$ & $33(3,8)$ & $\begin{array}{c}45,6 \\
(12,1)\end{array}$ & 2862 & 6 & 80 \\
\hline $\begin{array}{c}\text { Pellegrini, et } \\
\text { al. }{ }^{25}\end{array}$ & $\begin{array}{l}\text { Homens } \\
\text { e } \\
\text { Mulheres }\end{array}$ & $33,7(3,6)$ & $\begin{array}{l}44,2 \\
(8,7)\end{array}$ & 51 & 6 & 27 \\
\hline $\begin{array}{c}\text { Patrick,et } \\
a l .{ }^{24}\end{array}$ & Homens & $*$ & $43,9(8)$ & 441 & 12 & 30 \\
\hline
\end{tabular}




\begin{tabular}{|c|c|c|c|c|c|c|}
\hline Autores & Sexo & $\begin{array}{c}\text { IMC } \\
\text { médio } \\
(\mathrm{kg} / \mathrm{m} 2)\end{array}$ & $\begin{array}{l}\text { Idade } \\
\text { média }\end{array}$ & $\begin{array}{l}\text { Tamanho } \\
\text { da amostra }\end{array}$ & $\begin{array}{l}\text { Tempo de } \\
\text { seguimento } \\
\text { (meses) }\end{array}$ & $\begin{array}{c}\text { Perda de } \\
\text { seguimento } \\
(\%)\end{array}$ \\
\hline $\begin{array}{c}\text { Collins, et } \\
\text { al. }{ }^{34}\end{array}$ & $\begin{array}{l}\text { Homens } \\
\text { e } \\
\text { Mulheres }\end{array}$ & * & $*$ & 209 & 3 & 16 \\
\hline $\begin{array}{c}\text { Van } \\
\text { Genugten, } \\
\text { et al. }{ }^{29}\end{array}$ & $\begin{array}{l}\text { Homens } \\
\text { e } \\
\text { Mulheres }\end{array}$ & $28(1,94)$ & $\begin{array}{l}47,8 \\
(9,4)\end{array}$ & 539 & 6 & 35 \\
\hline $\begin{array}{c}\text { Chambliss, } \\
\text { et al. }{ }^{30}\end{array}$ & $\begin{array}{l}\text { Homens } \\
\text { e } \\
\text { Mulheres }\end{array}$ & * & $*$ & 120 & 3 & 21 \\
\hline $\begin{array}{l}\text { Booth, et } \\
\quad \text { al. }{ }^{31}\end{array}$ & $\begin{array}{l}\text { Homens } \\
\text { e } \\
\text { Mulheres }\end{array}$ & $30,6(*)$ & $47,5(*)$ & 73 & 3 & 27 \\
\hline Tate, et al. ${ }^{35}$ & $\begin{array}{l}\text { Homens } \\
\text { e } \\
\text { Mulheres }\end{array}$ & $*$ & $*$ & 192 & 6 & 19 \\
\hline $\begin{array}{c}\text { Mouttapa, et } \\
\text { al. }{ }^{22}\end{array}$ & Mulheres & $*$ & $*$ & 307 & 2 & 15 \\
\hline
\end{tabular}

*Autor não descreve IMC e idade médios e desvios-padrão totais da amostra.

Categoria 2: Desenho da intervenção e grupo controle, referencial teórico, estratégias de personalização e desfechos (tabela 2)

O desenho dos estudos de McConnon et al..$^{27}$, Patrick et al. ${ }^{24}$, Collins et al. ${ }^{34}$, Chambliss et al..$^{30} \mathrm{e}$ Tate et al. ${ }^{35}$ compararam um grupo que participava de programas de redução de peso online com um grupo controle que não recebia nenhuma orientação. Já os estudos de Gold et al. ${ }^{28}$, Rothert et al., ${ }^{26}$ Van Genugten et al. ${ }^{29}$ e Booth et al. ${ }^{31}$ realizaram um comparativo entre dois programas online, buscando implementar melhorias nos programas originais dos autores.

Os estudos de Morgan et al. ${ }^{23} \mathrm{e}$ Webber et al..$^{21}$ avaliaram as consultas presenciais realizadas por terapeutas, nutricionistas ou médicos versus programas online. Harvey-Berino et al., ${ }^{32}$ Polzien et al. ${ }^{33}$ e Pellegrini et al. ${ }^{25}$ compararam os resultados obtidos em três grupos: o tratamento presencial, os programas online e essas duas estratégias somadas. 
Os modelos utilizados na educação e intervenção, na sua maioria, foram baseados em teorias do comportamento. As teorias mais citadas foram: terapia cognitivo-comportamental, ${ }^{23,35}$ cognitivosocial ${ }^{22,24,33,34,}$ e teorias motivacionais. ${ }^{24,31}$

A maior parte das estratégias de personalização da intervenção foi elaborada a partir de informações coletadas por meio de questionários ou registros periódicos do peso corporal, consumo alimentar e frequência de atividade física, preenchidos pelos usuários no site. A partir dessas informações, o aconselhamento era elaborado pelos investigadores, que utilizavam os dados em consultas presenciais, retornos via correio eletrônico ou por sistemas específicos capazes de processar todos os dados e elaborar uma recomendação computadorizada.

O aconselhamento gerado pelo sistema de intervenção nutricional computadorizado era apresentado em forma de metas, dicas, textos e gráficos. Os assuntos abordados tinham relação com o grau de motivação do usuário, mudança do comportamento, alimentação, gastronomia e esporte.

Os estudos de Harvey-Berino et al. ${ }^{32}$, Webber et al. ${ }^{21}$ e Gold et al. ${ }^{28}$ utilizaram um sistema de intervenção nutricional computadorizado que possibilitava ao usuário comunicar-se com o investigador e com os demais participantes. Essa comunicação era estabelecida em salas de batepapo disponíveis periodicamente na página eletrônica do programa ou em blogs.

A redução no peso corporal alcançada variou de 0,9 a 8,8 Kg. Os trabalhos de Polzien et al. ${ }^{33}$ Gold et al., ${ }^{28}$ Rothert et al., ${ }^{26}$ Pellegrini et al., ${ }^{25}$ Patrick et al., ${ }^{24}$ Collins et al.,${ }^{34}$ Tate et al. ${ }^{35}$ e Mouttapa et al. ${ }^{22}$ apresentaram diferença estatisticamente significativa $(\mathrm{p}<0,05)$ na redução do peso, em comparação com o grupo controle. Dois trabalhos de seguimento mais longo mostraram diferença significativa na manutenção do peso após sua redução, em um período de 12 meses. ${ }^{24,28}$

Determinados artigos demonstram redução de peso clinicamente significativa (> 5\%) entre os participantes. Nos estudos de McConnon et al., ${ }^{27}$ Morgan et al., ${ }^{23}$ Collins et al. ${ }^{34}$ e Tate et al.,${ }^{35}$ o percentual de redução acima ou igual a $5 \%$ do peso corporal entre os participantes foi maior do que no grupo controle. 
Tabela 2. Desenho da intervenção e grupo controle, referencial teórico, estratégias de personalização e desfechos. São Paulo-SP, 2012.

\begin{tabular}{|c|c|c|}
\hline \multicolumn{3}{|l|}{ Autores } \\
\hline \multirow[t]{4}{*}{$\begin{array}{l}\text { Mc } \\
\text { Connon } \\
\text { et al. }{ }^{27}\end{array}$} & $\begin{array}{l}\text { Desenho grupo } \\
\text { controle e } \\
\text { intervenção }\end{array}$ & $\begin{array}{l}\text { Grupo controle: tratamento usual } \\
\text { Grupo intervenção: internet com acessos livres ao site }\end{array}$ \\
\hline & $\begin{array}{l}\text { Referencial } \\
\text { teórico } \\
\text { comportamental }\end{array}$ & Não descreve \\
\hline & $\begin{array}{l}\text { Estratégias de } \\
\text { personalização }\end{array}$ & $\begin{array}{l}\text { O site promovia ferramentas de apoio para mudança de } \\
\text { comportamento. Mensagens motivacionais foram geradas com } \\
\text { base no relatório dos participantes.. }\end{array}$ \\
\hline & Desfecho & $\begin{array}{l}\text { Redução média do peso: Grupo Internet: } 1,3 \mathrm{~kg} \text {, Grupo controle } \\
1,9 \mathrm{~kg}(\mathrm{p}=0,56) . \\
\text { Perda de peso > } \mathbf{5 \%} \text { : Grupo Internet } 22 \% \text {; Grupo controle } 18 \%\end{array}$ \\
\hline \multirow[t]{4}{*}{$\begin{array}{l}\text { Morgan } \\
\text { et al. }{ }^{23}\end{array}$} & $\begin{array}{l}\text { Desenho grupo } \\
\text { controle e } \\
\text { intervenção }\end{array}$ & $\begin{array}{l}\text { Grupo controle consulta presencial + manual para redução do } \\
\text { peso. Grupo intervenção de consulta presencial (orientações sobre } \\
\text { o uso do site) + registro do peso semanal, diário atividade física e } \\
\text { alimentar no site }\end{array}$ \\
\hline & $\begin{array}{l}\text { Referencial } \\
\text { teórico } \\
\text { comportamental }\end{array}$ & No grupo intervenção: Teoria cognitivo-comportamental \\
\hline & $\begin{array}{l}\text { Estratégias de } \\
\text { personalização }\end{array}$ & $\begin{array}{l}\text { Feedbacks baseados nos registros alimentares, de peso e exercício } \\
\text { físico foram enviados por sete vezes. Os usuários podiam postar } \\
\text { mensagens na página eletrônica do programa }\end{array}$ \\
\hline & Desfecho & $\begin{array}{l}\text { Redução média do peso: O grupo Internet } 5,3 \mathrm{~kg} \text {, Grupo controle } \\
3,1 \mathrm{~kg}(\mathrm{p}=0.408) \\
\text { Perda de peso > 5\%: Grupo Internet: } 57,7 \% \text {; Grupo control e: } \\
30 \%(\mathrm{p}=0.062)\end{array}$ \\
\hline $\begin{array}{l}\text { Harvey- } \\
\text { Berino, } \\
\text { et al. }{ }^{32}\end{array}$ & $\begin{array}{l}\text { Desenho grupo } \\
\text { controle e } \\
\text { intervenção }\end{array}$ & $\begin{array}{l}\text { Grupo: presencial: encontro semanal em grupo + controle } \\
\text { calórico } \\
\text { Grupo Internet: web + encontros mensais online em grupo (chat) + } \\
\text { controle calórico } \\
\text { Grupo misto: características do grupo internet + encontros } \\
\text { presenciais em grupo uma vez por mês }+ \text { controle calórico }\end{array}$ \\
\hline
\end{tabular}




\begin{tabular}{|c|c|c|}
\hline & $\begin{array}{l}\text { Referencial } \\
\text { teórico } \\
\text { comportamental }\end{array}$ & $\begin{array}{l}\text { Nos três grupos: Utilização de estratégias comportamentais e } \\
\text { autoadministração de competências }\end{array}$ \\
\hline & $\begin{array}{l}\text { Estratégias de } \\
\text { personalização }\end{array}$ & $\begin{array}{l}\text { Estratégias comportamentais incluíam automonitoramento, } \\
\text { controle de estímulos, resolução de problemas, definição de metas, } \\
\text { prevenção de recaídas, e treinamento de assertividade }\end{array}$ \\
\hline & Desfecho & $\begin{array}{l}\text { Redução média do peso: } O \text { grupo presencial } 8,0 \mathrm{~kg} \text {, Grupo } \\
\text { internet } 5,5 \mathrm{~kg} \text {; grupo misto } 6,0 \mathrm{Kg}(\mathrm{p}=0,01) \\
\text { Perda de peso > 7\%: grupo presencial } 56,3 \% \text {; grupo internet } \\
37,3 \% \text {; grupo misto 44,4\% (p } 0,01)\end{array}$ \\
\hline \multirow[t]{4}{*}{$\begin{array}{l}\text { Webber, } \\
\text { et al. }{ }^{21}\end{array}$} & $\begin{array}{l}\text { Desenho grupo } \\
\text { controle e } \\
\text { intervenção }\end{array}$ & $\begin{array}{l}\text { Grupos controle: } \\
\text { Intervenção web mínima: } \\
\text { Grupo Intervenção web avançada: mesma estratégia do grupo } \\
\text { anterior + sala de bate papo. }\end{array}$ \\
\hline & $\begin{array}{l}\text { Referencial } \\
\text { teórico } \\
\text { comportamental }\end{array}$ & Entrevista motivacional \\
\hline & $\begin{array}{l}\text { Estratégias de } \\
\text { personalização }\end{array}$ & $\begin{array}{l}\text { O site era atualizado com: dicas para redução do peso, exercícios } \\
\text { semanais, diário para automonitoramento, links para outros sites e } \\
\text { havia uma plataforma para mensagens. } \\
\text { As salas de bate papo utilizavam técnicas motivacionais }\end{array}$ \\
\hline & Desfecho & $\begin{array}{l}\text { Redução média do peso: O grupo Intervenção web mínima 2,22 } \\
\text { kg, Grupo Intervenção web avançada: } 3,71 \mathrm{~kg}(\mathrm{p}=0,19) \\
\text { Perda de peso > 5\%: } \mathbf{6 7 \%} \text { grupo Intervenção web mínima e } 46 \% \\
\text { grupo Intervenção web avançada }(\mathrm{p}=0,07)\end{array}$ \\
\hline \multirow[t]{2}{*}{$\begin{array}{l}\text { Polzien, } \\
\text { et al. }{ }^{33}\end{array}$} & $\begin{array}{l}\text { Desenho grupo } \\
\text { controle e } \\
\text { intervenção }\end{array}$ & $\begin{array}{l}\text { Grupo controle presencial: recebiam sete consultas no primeiro } \\
\text { mês, duas no segundo e uma no terceiro } \\
\text { Grupo misto: (presencial + tecnologia). presencial + um monitor } \\
\text { para medição do gasto energético que deveria ser utilizado três } \\
\text { vezes. Grupo tecnologia: presencial + um monitor para controle } \\
\text { do gasto energético para uso diário }\end{array}$ \\
\hline & $\begin{array}{l}\text { Referencial } \\
\text { teórico } \\
\text { comportamental }\end{array}$ & Teoria cognitiva social \\
\hline
\end{tabular}




\begin{tabular}{|c|c|c|}
\hline & $\begin{array}{l}\text { Estratégias de } \\
\text { personalização }\end{array}$ & $\begin{array}{l}\text { O sistema capaz de monitorar o gasto energético fornecia feedback } \\
\text { entre o balanço energético. } \\
\text { No final do dia, cada usuário deveria fazer o upload pelo sistema } \\
\text { no site do programa. } \\
\text { Os investigadores checavam o balanço energético para realização } \\
\text { do feed back nas consultas presenciais }\end{array}$ \\
\hline & Desfecho & $\begin{array}{l}\text { Redução média do peso: Grupo controle presencial 4,1 kg, Grupo } \\
\text { misto: } 3,4 \mathrm{~kg} \text {, Grupo tecnologia: } 6,2 \mathrm{Kg} \\
(\mathrm{p}=0,04) \\
\text { Perda de peso > 5\%: não descreve }\end{array}$ \\
\hline \multirow[t]{4}{*}{$\begin{array}{l}\text { Gold, } \\
\text { et al. }{ }^{28}\end{array}$} & $\begin{array}{l}\text { Desenho grupo } \\
\text { controle e } \\
\text { intervenção }\end{array}$ & $\begin{array}{l}\text { Grupo intervenção web comportamental (seis meses redução de } \\
\text { peso e seis meses manutenção do peso). } \\
\text { Grupo intervenção web comercial }\end{array}$ \\
\hline & $\begin{array}{l}\text { Referencial } \\
\text { teórico } \\
\text { comportamental }\end{array}$ & Estratégias do comportamento alimentar \\
\hline & $\begin{array}{l}\text { Estratégias de } \\
\text { personalização }\end{array}$ & $\begin{array}{l}\text { Período de redução de peso: } \\
\text { Os usuários deveriam acessar o site e registrar o peso e consumo } \\
\text { alimentar e responder algumas questões relacionadas ao } \\
\text { comportamento alimentar e peso. Havia feedback sobre as resposta } \\
\text { enviado para o email do usuários. } \\
\text { Havia sala de bate papo e blogs }\end{array}$ \\
\hline & Desfecho & $\begin{array}{l}\text { Redução média do peso em } 12 \text { meses: Grupo intervenção web } \\
\text { comportamental: } 7,8 \mathrm{Kg} \text {, Grupo intervenção web comercial: } 3,4 \mathrm{~kg} \\
(\mathrm{p}=0,002) \\
\text { Perda de peso > 5\%: Grupo intervenção web comportamental: } \\
65 \% \text {, Grupo intervenção web comercial: } 37.5 \% \text {; }(p=0.01) \text { em } 12 \\
\text { meses }\end{array}$ \\
\hline \multirow[t]{2}{*}{$\begin{array}{l}\text { Rothert, } \\
\text { et al. }{ }^{26}\end{array}$} & $\begin{array}{l}\text { Desenho grupo } \\
\text { controle e } \\
\text { intervenção }\end{array}$ & $\begin{array}{l}\text { Grupo } 1 \text { informação usual via web para redução de peso } \\
\text { Grupo } 2 \text { sistema via web personalizado desenvolvido para redução } \\
\text { de peso }\end{array}$ \\
\hline & $\begin{array}{l}\text { Referencial } \\
\text { teórico } \\
\text { comportamental }\end{array}$ & Não descreve \\
\hline
\end{tabular}




\begin{tabular}{|c|c|c|}
\hline & $\begin{array}{l}\text { Estratégias de } \\
\text { personalização }\end{array}$ & $\begin{array}{l}\text { Um questionário era aplicado para os dois grupos no baseline. Não } \\
\text { foi prescrita dieta, os pacientes recebiam feedback automático de } \\
\text { acordo com as informações coletadas no questionário. O suporte } \\
\text { social também era realizado entre os usuários por meio de blog }\end{array}$ \\
\hline & Desfecho & $\begin{array}{l}\text { Redução média do peso: Grupo 1: 2,8kg, Grupo 2: 1,1 Kg } \\
(\mathrm{p}=0,0007) \\
\text { Perda de peso > 5\%: não descreve }\end{array}$ \\
\hline \multirow[t]{4}{*}{$\begin{array}{l}\text { Pellegrini, } \\
\text { et al. }{ }^{25}\end{array}$} & $\begin{array}{l}\text { Desenho grupo } \\
\text { controle e } \\
\text { intervenção }\end{array}$ & $\begin{array}{l}\text { Grupo intervenção presencial. } \\
\text { Grupo misto: (presencial + tecnologia). Grupo tecnologia: monitor } \\
\text { do gasto energético + correios eletrônicos dos investigadores + } \\
\text { ligações telefônicas uma vez por mês. }\end{array}$ \\
\hline & $\begin{array}{l}\text { Referencial } \\
\text { teórico } \\
\text { comportamental }\end{array}$ & Estratégias comportamentais \\
\hline & $\begin{array}{l}\text { Estratégias de } \\
\text { personalização }\end{array}$ & $\begin{array}{l}\text { Reuniões mensais sendo uma presencial e três em grupo nas } \\
\text { semanas } \\
\text { Os usuários do monitor utilizavam durante o dia e descarregavam } \\
\text { as informações no site do programa onde registravam o consumo } \\
\text { alimentar e o peso. Os investigadores avaliavam um feedback } \\
\text { semanal. }\end{array}$ \\
\hline & Desfecho & $\begin{array}{l}\text { Redução média do peso: Grupo intervenção presencial: } 7,1 \mathrm{~kg} \text {; } \\
\text { Grupo misto: } 8,8 \mathrm{~kg} \text {; Grupo tecnologia: } 7,6 \mathrm{~kg} \\
(\mathrm{p}=0,001) \\
\text { Perda de peso > 5\%: não descreve }\end{array}$ \\
\hline \multirow[t]{3}{*}{$\begin{array}{l}\text { Patrick, } \\
\text { et al. }{ }^{24}\end{array}$} & $\begin{array}{l}\text { Desenho grupo } \\
\text { controle e } \\
\text { intervenção }\end{array}$ & $\begin{array}{l}\text { Grupo intervenção: programa de redução de peso online } \\
\text { Grupo controle: hábito usual }\end{array}$ \\
\hline & $\begin{array}{l}\text { Referencial } \\
\text { teórico } \\
\text { comportamental }\end{array}$ & Teoria cognitivo-social e modelo determinante do comportamento \\
\hline & $\begin{array}{l}\text { Estratégias de } \\
\text { personalização }\end{array}$ & $\begin{array}{l}\text { Um grupo focal foi realizado com alguns homens antes do início } \\
\text { do programa. As informações coletadas neste grupo foram } \\
\text { utilizadas para adaptar o site a realidade da ótica masculina na } \\
\text { redução do peso. }\end{array}$ \\
\hline
\end{tabular}




\begin{tabular}{|c|c|c|}
\hline & Desfecho & $\begin{array}{l}\text { Redução média do peso: Grupo intervenção: } 0,9 \mathrm{~kg} \text { Grupo } \\
\text { controle: } 0,2 \mathrm{~kg}(\mathrm{p}=0,01) \\
\text { Perda de peso > } \mathbf{5 \%} \text { : não se aplica }\end{array}$ \\
\hline \multirow[t]{4}{*}{$\begin{array}{l}\text { Collins, } \\
\text { et al. }{ }^{34}\end{array}$} & $\begin{array}{l}\text { Desenho grupo } \\
\text { controle e } \\
\text { intervenção }\end{array}$ & $\begin{array}{l}\text { Grupo controle: lisa de espera; Grupo intervenção web online } \\
\text { padrão; Grupo intervenção web online avançada }\end{array}$ \\
\hline & $\begin{array}{l}\text { Referencial } \\
\text { teórico } \\
\text { comportamental }\end{array}$ & $\begin{array}{l}\text { Teoria cognitivo-social e mediadores-chave da mudança } \\
\text { comportamental }\end{array}$ \\
\hline & $\begin{array}{l}\text { Estratégias de } \\
\text { personalização }\end{array}$ & $\begin{array}{l}\text { Recomendação utilizar quatro vezes na semana e registrar o } \\
\text { consumo alimentar e atividade física. O registro do peso era } \\
\text { diário. } \\
\text { O programa avançado incluía metas de peso e mudanças no } \\
\text { comportamento. }\end{array}$ \\
\hline & Desfecho & $\begin{array}{l}\text { Redução média do peso: Grupo intervenção web padrão: 2,1 kg; } \\
\text { Grupo intervenção web avançada: } 3,0 \mathrm{~kg} \\
\text { Grupo controle: } 0,4 \mathrm{~kg}(\mathrm{p}=0,001) \\
\text { Perda de peso > 5\%: Grupo intervenção web padrão: } 18 \% \text {; Grupo } \\
\text { intervenção web avançada: } 17 \% \text {; Grupo controle: } 3 \%\end{array}$ \\
\hline \multirow[t]{4}{*}{$\begin{array}{l}\text { Van } \\
\text { Genugten, } \\
\text { et al. }{ }^{29}\end{array}$} & $\begin{array}{l}\text { Desenho grupo } \\
\text { controle e } \\
\text { intervenção }\end{array}$ & $\begin{array}{l}\text { Grupo controle: informação via web generalizada; Grupo } \\
\text { intervenção: personalizada via web }\end{array}$ \\
\hline & $\begin{array}{l}\text { Referencial } \\
\text { teórico } \\
\text { comportamental }\end{array}$ & $\begin{array}{l}\text { Intervention Mapping approach; } \\
\text { Self-regulation theory; } \\
\text { Theory of Planned Behavior; } \\
\text { Precaution Adoption Process Model }\end{array}$ \\
\hline & $\begin{array}{l}\text { Estratégias de } \\
\text { personalização }\end{array}$ & $\begin{array}{l}\text { Visitar o site três a quatro vezes em um período de dois meses. A } \\
\text { cada duas semanas, recebiam um lembrete para acessar o sitio. }\end{array}$ \\
\hline & Desfecho & $\begin{array}{l}\text { Redução média do peso: Não descreve redução média de peso } \\
\text { Perda de peso > } 5 \% \text { : Não descreve redução do peso em \% }\end{array}$ \\
\hline $\begin{array}{l}\text { Chambliss, } \\
\text { et al. }{ }^{30}\end{array}$ & $\begin{array}{l}\text { Desenho grupo } \\
\text { controle e } \\
\text { intervenção }\end{array}$ & $\begin{array}{l}\text { Grupo Básico: sistema computadorizado para redução de peso } \\
\text { com feedback. Grupo avançado: sistema computadorizado para } \\
\text { redução de peso com com feedback voltado para estratégias do } \\
\text { comportamento. Grupo hábito usual }\end{array}$ \\
\hline
\end{tabular}




\begin{tabular}{|c|c|c|}
\hline & $\begin{array}{l}\text { Referencial } \\
\text { teórico } \\
\text { comportamental }\end{array}$ & Não descreve \\
\hline & $\begin{array}{l}\text { Estratégias de } \\
\text { personalização }\end{array}$ & $\begin{array}{l}\text { Grupo básico: plano dietético personalizado, automonitoramento } \\
\text { diário do consumo alimentar e atividade física pelo software. } \\
\text { Semanalmente os educadores enviavam feedback com base no } \\
\text { consumo alimentar e atividade física. Todo mês havia uma } \\
\text { consulta presencial. O grupo avançado recebia as mesmas } \\
\text { informações do outro grupo, mas incluía estratégia para } \\
\text { manejo do comportamento alimentar, uma vez por mês boletins } \\
\text { informativos, uma ligação telefônica mensal }\end{array}$ \\
\hline & Desfecho & $\begin{array}{l}\text { Redução média do peso: Grupo básico 2,7 Kg Grupo avançado } \\
2,5 \mathrm{~kg} \text { Grupo controle }+0,3,(\mathrm{p}=0,61) \\
\text { Perda de peso > 5\%: Grupo básico 52\%; Grupo avançado } 35 \% \text {; } \\
\text { Grupo controle }\end{array}$ \\
\hline \multirow[t]{4}{*}{$\begin{array}{l}\text { Booth, } \\
\text { et al. }{ }^{31}\end{array}$} & $\begin{array}{l}\text { Desenho grupo } \\
\text { controle e } \\
\text { intervenção }\end{array}$ & $\begin{array}{l}\text { Grupo ED: intervenção dietética e física via web } \\
\text { Grupo Ex: intervenção somente com orientação de exercício físico } \\
\text { via web }\end{array}$ \\
\hline & $\begin{array}{l}\text { Referencial } \\
\text { teórico } \\
\text { comportamental }\end{array}$ & Teoria motivacional: "goal setting theory" \\
\hline & $\begin{array}{l}\text { Estratégias de } \\
\text { personalização }\end{array}$ & $\begin{array}{l}\text { Descrição do grupo com tratamento dietético: os indivíduos } \\
\text { respondiam questões sobre o consumo de alguns alimentos. A } \\
\text { partir disto, deveriam estipular metas para melhorar o consumo } \\
\text { de certos alimentos. No mínimo uma e no máximo } 12 \text { metas } \\
\text { deveriam ser preenchidas por semana. O mesmo ocorria com a } \\
\text { ingestão calórica. Um retorno positivo era enviado ao usuário, } \\
\text { assim como estratégias, para identificar barreiras de mudança. } \\
\text { Recebiam ainda boletins informativos sobre nutrição em geral }\end{array}$ \\
\hline & Desfecho & $\begin{array}{l}\text { Redução média do peso: Grupo ED: } 0,7 \mathrm{Kg} \text { Grupo Ex: } 1,9 \mathrm{~kg} \\
(\mathrm{p}=0,09) \\
\text { Perda de peso }>\mathbf{5 \%} \text { : Não se aplica }\end{array}$ \\
\hline $\begin{array}{l}\text { Tate, } \\
\text { et al. }\end{array}$ & $\begin{array}{l}\text { Desenho grupo } \\
\text { controle e } \\
\text { intervenção }\end{array}$ & $\begin{array}{l}\text { Grupo intervenção via web com feedback automático; grupo } \\
\text { intervenção via web elaborado por um profissional } \\
\text { Grupo controle: hábito usual }\end{array}$ \\
\hline
\end{tabular}




\begin{tabular}{|c|c|c|}
\hline & $\begin{array}{l}\text { Referencial } \\
\text { teórico } \\
\text { comportamental }\end{array}$ & Teoria cognitivo-comportamental \\
\hline & $\begin{array}{l}\text { Estratégias de } \\
\text { personalização }\end{array}$ & $\begin{array}{l}\text { Dieta }(1.200 \text { a } 1.500 \mathrm{kcal})+\text { atividade física no baseline. No site, } \\
\text { estímulo ao registro do peso semanal, com respostas automáticas } \\
\text { em forma de gráficos e dicas para redução do consumo calórico e } \\
\text { mudança do comportamento. }\end{array}$ \\
\hline & Desfecho & $\begin{array}{l}\text { Redução média do peso: Grupo intervenção via web com feedback } \\
\text { automático; 4,9 Kg; Grupo intervenção via web elaborado por um } \\
\text { profissional : 7,3kg; Grupo controle: } 2,6 \mathrm{~kg} \text {; }(\mathrm{p}=0,001) \\
\text { Perda de peso > 5\%: Grupo intervenção via web com feedback } \\
\text { automático; } 34 \% \text {; Grupo intervenção via web elaborado por um } \\
\text { profissional : } 52 \% \text {; Grupo controle: } 27 \%(\mathrm{p}=0,01)\end{array}$ \\
\hline \multirow[t]{4}{*}{$\begin{array}{l}\text { Mouttapa, } \\
\text { et al. }{ }^{22}\end{array}$} & $\begin{array}{l}\text { Desenho grupo } \\
\text { controle e } \\
\text { intervenção }\end{array}$ & $\begin{array}{l}\text { Grupo intervenção - programa de intervenção nutricional via } \\
\text { web; } \\
\text { Grupo controle - sem intervenção }\end{array}$ \\
\hline & $\begin{array}{l}\text { Referencial } \\
\text { teórico } \\
\text { comportamental }\end{array}$ & Teoria cognitiv- social \\
\hline & $\begin{array}{l}\text { Estratégias de } \\
\text { personalização }\end{array}$ & $\begin{array}{l}\text { Intervenção nutricional individualizada. } \\
\text { O participante elegia uma meta redução de peso ou prevenção } \\
\text { das doenças crônicas e ainda eram estimulados a identificar quais } \\
\text { seriam as próximas sete metas realistas. }\end{array}$ \\
\hline & Desfecho & $\begin{array}{l}\text { Redução média do peso: Grupo intervenção: } 0,885 \mathrm{~kg} \text {; Grupo } \\
\text { controle: } 0,227 \mathrm{~kg}(\mathrm{p}=0,05) \\
\text { Perda de peso > } \mathbf{5 \%} \text { : não descreve }\end{array}$ \\
\hline
\end{tabular}




\section{Discussão}

Os estudos de Polzien et al., ${ }^{33}$ Gold et al., ${ }^{28}$ Rothert et al., ${ }^{26}$ Pellegrini et al., ${ }^{25}$ Patrick et al. ${ }^{24}$ Collins et al., ${ }^{34}$ Tate et al. ${ }^{35}$ e Moutappa et al. ${ }^{22}$ relataram diferença estatisticamente significativa $(\mathrm{p}<0,05)$ na redução do peso no grupo intervenção. Todavia, os resultados nesta revisão, assim como na de Arem et al., ${ }^{36}$ sugerem que intervenções via web não promovem um efeito uniforme na redução do peso porque dependem do desenho de estudo, do tempo de seguimento da intervenção e da adesão ao tratamento. Com relação à faixa de redução de peso, não foi possível realizar uma análise estatística para constatar se houve diferença entre os estudos, pois as metodologias (tamanho amostral, tempo de seguimento e esquema de tratamento) eram diferentes.

Uma metanálise $^{37}$ de 18 estudos publicada em 2009 sugeriu que intervenções via web promovem redução do peso similar nos grupos com intervenção mínima. Aparentemente, só é possível alcançar efeito significativo no peso corporal quando o programa permite interações entre indivíduo, comunidade, profissional da saúde e o website. Nesta revisão, os estudos que apresentaram diminuição satisfatória no peso permitiam tais interações e, adicionalmente, nos estudos de Polzien et al. ${ }^{33}$ e Pellegrini et al., ${ }^{25}$ foi utilizado um aparelho que monitorava o gasto energético durante 24 horas e ficava acoplado ao braço do indivíduo. No final do dia, o participante deveria acessar a página online do programa e a atualizá-lo com as informações do monitor.

Contento $^{38}$ discutiu que a educação nutricional focada na redução do peso tem maior probabilidade de ser eficaz quando é realizada presencialmente, se estiver adequada às necessidades individuais e comportamentais e se estabelecer metas, estratégias, fornecer feedback durante o tratamento e possibilitar a participação ativa do indivíduo no processo de aprendizagem. Nesta revisão, assim como na de Enwald \& Huotari, ${ }^{39}$ foi possível observar que os componentes da intervenção via web que estão potencialmente associados com eficácia são aqueles que favorecem a personalização do tratamento e possuem as seguintes características:

- Melhor resultado relacionado ao maior número de acessos (logins) ao site pelo usuário;

- Disponibilidade de ferramentas de automonitoramento no programa, como: diários para registro alimentar e de atividade física;

- Suporte social, como comunicação entre os usuários;

- Atendimento online (chat ou salas de bate papo) com troca de mensagens com profissionais nutricionistas ou psicólogos. 
A literatura discute que a melhor adesão às orientações está relacionada com o estado motivacional do indivíduo. Os programas via web podem auxiliar nesse despertar para a intenção de mudança: por exemplo, ao mostrar para o participante como está seu hábito alimentar atual, uma vez que possibilitam o cálculo do consumo dietético e sua comparação instantânea com as recomendações dietéticas. ${ }^{18}$

Além do grau de motivação do indivíduo, o sexo é outra característica que pode influenciar no sucesso da intervenção. Dentre os artigos selecionados nesta revisão, os trabalhos de Morgan et al., ${ }^{23}$ Webber et al., ${ }^{21}$ Patrick et al. ${ }^{24}$ e Moutappa et al..${ }^{22}$ foram desenvolvidos especificamente para mulheres ou homens. No estudo de Patrick et al., ${ }^{24}$ por exemplo, um grupo focal foi realizado com alguns homens antes do início do programa e as informações coletadas foram utilizadas para adaptar o programa sob a ótica masculina no contexto da redução do peso.

Além disso, mulheres e homens podem ter preferências distintas pelo tipo de intervenção. No estudo de Terry et al., ${ }^{40}$ em que os participantes podiam escolher entre as intervenções via web ou telefônica, os indivíduos do sexo masculino preferiram a primeira opção.

\section{Conclusão}

Os atuais sistemas da web proporcionam interação e praticidade, podendo ser acessados quantas vezes forem necessárias, a qualquer hora e em qualquer lugar. Além disso, há participação ativa do indivíduo, representada pela interatividade entre programa/indivíduo e programa/grupo (redes sociais), que estimula o engajamento do participante no processo de emagrecimento. Dessa maneira, pode-se induzir o despertar para o processo de mudança do estilo de vida.

Os estudos controlados com intervenções via web são muito recentes e os recursos utilizados na intervenção, na maioria das vezes, não são descritos detalhadamente pelos autores, o que dificulta a criação de novos programas com evidência científica.

Além disso, os programas formulados para redução de peso via web promovem reduções de peso variadas, muitas vezes clinicamente não satisfatórias. Aqueles programas que contribuíram para redução de peso maior do que $5 \%$ do peso inicial não possuem similaridade no modelo de intervenção. 


\section{Referências}

1. World Health Organization. Diet, nutrition and the prevention of chronic diseases. Geneva: WHO; 2003. WHO technical report series; 916.

2. World Health Organization. Obesity and overweight. Geneva: WHO; 2012.

3. Brasil. Ministério da Saúde. Vigitel Brasil 2011: Vigilância de fatores de risco e Proteção para doenças crônicas por inquérito telefônico. Brasília: Ministério da Saúde; 2012.

4. World Health Organization. Action plan for the global strategy for the prevention and control of non-communicable diseases. Geneva: WHO; 2010.

5. Prentice AM, Jebb SA. Obesity in Britain: gluttony or sloth? BMJ. 1995; 311:437-39.

6. Hawkes C, Blouin C, Henson S, Drager N, Dubé L. Trade, food, diet and health: perspectives and policy options. Oxford: Wiley Blackwell; 2010.

7. Monteiro CA, Mondini L, Souza AL, Popkin B. Da desnutrição para a obesidade: a transição nutricional no Brasil. In: Monteiro CA, Velhos e Novos Males da Saúde no Brasil: a evolução do país e suas doenças. São Paulo: Hucitec; 2000. p. 267-55.

8. Associação Brasileira para o Estudo de obesidade e da Síndrome Metabólica. Diretrizes brasileiras de obesidade 2009/2010. $3^{\circ}$ ed. Itapevi, SP: Ac Farmacêutica, 2009.

9. Karlsen TI, Sohagen M, Hjelmesaeth J. Predictors of weight loss after an intensive lifestyle intervention program in obese patients: a 1 year prospective cohort study. Health and Quality of Life Outcomes. 2013; 11:165.

10. The Diabetes Prevention Program Research Group. Effect of weight loss with lifestyle on risk of diabetes. DPP. Diabetes Care. 2006; 29:2102-2107.

11. Tate DF, Finkelstein EA, Khavjou O, Gustafson A. Cost effectiveness of internet interventions: review and recommendations. Ann. Behav. Med. 2009; 38: 40-45

12. Arem H, Irwin M. A review of web-based weight loss interventions in adults. Obes Rev. 2011; 12(5):266-263.

13. Kroeze W, Werkman A, Brug J. A sistematic review of randomized trials on the effectivemess of computer-tailored education on physical activity and dietary behaviors. Ann. Behav. Med. 2006; 31(3):205-226.

14. Neve M, Morgan, PJ, Jones, PR, Collins E. Effectivenees of web-based interventions in achieving weight loss maitenace in overweight and obese adults: a systematic rewiew with meta-analysis. Obesity Reviews. 2010; 11:306-321.

15. Enwald HPK, Huotari MLA. Preventing the obesity epidemia by second generation tailored health communication: an. interdisciplinary review. J. Med. Internet Res. 2010; 12(2):e24.

16. Lustria MLA, Cortese J, Noar SM, Glueckauf RL. Computer-tailored health interventions delivered over the web: review and analysis of key components. Patient Education and Counselling. 2009; 74:156-173. 
17. Brug J, Oenema A, Kroeze W, Raat H. The internet and nutrition education: challenges and oportunities. Eur. J. Clin. Nutr. 2005; 59(Supl.1):S130-S7.

18. Tate DF. A series of studies examining internet treatment of obesity to inform internet interventions for substance use and misuse. Subs. Use Misuse. 2011; 46(1):57-65.

19. Comitê Gestor da Internet no Brasil. Pesquisa sobre o uso das tecnologias de informação e comunicação no Brasil: TIC Domicílios e TIC Empresas 2010 São Paulo: CGI.br, NIC.br, CETIC.br; 2011.

20. Santos LAS. Da dieta a reeducação alimentar: algumas notas sobre o comer contemporâneo a partir dos programas de emagrecimento na internet. Physis: Rev. Saúde Coletiva. 2010; 20(2):459-474.

21. Webber KH, Tate DF, Bowlimg M. A randomized comparison of two motivationally enhanced Internet behavioral weight loss programs. Behavior Research and Therapy. 2008; 46:1090-1095.

22. Mouttapa M, Robertson TP, McEligot AJ, Weiss JW, Hoolihan L, Ora A, Trinh L. The personal nutrition planner: a 5-week, computer-tailored intervention for women. J. Nutr. Educ. Behav. 2011; 43(3):165-172.

23. Morgan PJ, Lubanst DR, Collins CE, Warren JM, Callist R. 12-month outcomes and process evaluation of the shed-it rct: an internet-based weight loss program targeting men. Obesity. 2011; 19(11):142-152.

24. Patrick K, Calfas KJ, Norman GJ, Rosenberg D, Zabinski MF, Sallis JF, Rock CL, Dillon LW. Outcomes of a 12-month web-based intervention for overweight and obese men. Ann. Behav. Med. 2011; 42:391-401.

25. Pellegrini CA, Verba SD, Otto Ad, Helse DL, Davis KK, Jakicic JM. The Comparison of a technologybased system and an in-person behavioral weight loss intervention. Obesity. 2012; 20(2):356-63.

26. Rothert K, Strecher VJ, Doyle LA, Caplan WM, Joyce JS, Jimison HB, Karm LM, Mims AD, Roth MA, Web-based weight management programs in an integrated health care setting: a randomized, controlled trial. Obesity. 2006; 14(2):277-272.

27. McConnon A, Kirk SFL, Cockroft JE, Harvey EL, Greenwood DC, Thomas JD, Ransley JK, Bojke L. The Internet for weight control in an obese sample: results of a randomised controlled trial. BMC Health Services Research. 2007; 7:206.

28. Gold BC, Burke S, Pintauro S, Buzzel P, Harvey-Berino J. Weight loss on the web: a pilot study comparing a structured behavioral intervention to a commercial program. Obesity. 2007; 15(1):155-165.

29. Van Genugten L, Van Empelen P, Boon B, Borsboom G, Visscher, Oenema A. Results from an online computer-tailored weight management intervention for overweight adults: randomized controlled Trial. J. Med. Internet Res. 2012; 14(2):e44.

30. Chambliss HO, Huber RC, Finley CE, McDoniel SO, Kitzman-Ulrich H, Wilkinson WJ. Computerized self-monitoring and technology-assisted feedback for weight loss with and without an enhanced behavioral component. Patient Educ. Couns. 2011; 85(3):375-82.

31. Booth AO, Nowson CA, Matters H. Evaluation of an interactive, internet-based weight loss program: a pilot study. Health Education Research. 2008; 23(3):372-381. 
32. Harvey-Berino J, West D, Krukowski R, Prewitt E, VanBiervliet A, Ashikaga T, Skelly J. Internet delivered behavioral obesity treatment. Prev Med. 2010 51(2):123-128.

33. Polzien KM, Jakicic JM, Tate D, Otto A D. The Efficacy of a technology-based system in a shortterm behavioral weight loss intervention. 2007; 15(4):826-831.

34. Collins CE, Morgan PJ, Jones P, Fletcher K, Martin J, Aguiar EJ, Lucas A, et al. A 12-week commercial web-based weight-loss program for overweight and obese adults: randomized controlled trial comparing basic versus enhanced features. J. Med. Internet Res. 2012; 14(2):e57

35. Tate DF, Jackvony EH, Wing RR. A randomized trial comparing human e-mail counseling, computerautomated tailored counseling, and no counseling in an internet weight loss program. Arch. Intern. Med. 2006; 166(15):1620-5.

36. Arem H, Irwin M. A Review of web-based weight loss interventions in adults. Obes. Rev. 2011; 12(5):236-243.

37. Neve M, Morgan P J, Jones P R, Collins C E. Effectiveness of web-based interventions in achieving weight loss and weight loss maintenance in overweight and obese adults: a systematic review with meta-analysis. Obes. Rev. 2010; 11(4):306-321.

38. Contento IR. Nutrition education: linking research, theory, and practice. Asia Pac. J. Clin. Nutr. 2008;17(1):176-179

39. Enwald HPK, Huotari MA. Preventing the obesity epidemic by second generation tailored health communication: an interdisciplinary review. J. Med. Internet Res. 2010; 12(2):e24.

40. Terry PE, Seaverson ELD, Staufacker MJ, Gingerch SO. A comparison of effectiveness of a telephone coaching program and a mail-based program. Health Educ. Behav. 2010; 37(6):895-912.

Recebido: 23/9/2013

Revisado: 30/10/2013

Aprovado: 06/1/2014 